\title{
UNA INTERFAZ TRANSFRONTERIZA COMPLEJA. El caso del Golfo de Fonseca: El Salvador, Honduras y Nicaragua
}

\author{
A complexcross-border interface. The case of the Gulf of Fonseca: El Salvador, Honduras and Nicaragua. \\ Lucile Medina
}

Resumen: La región del Golfo de Fonseca es conocida en Centroamérica por las discrepancias en las delimitaciones fronterizas que han enfrentado secularmente los tres Estados ribereños - El Salvador, Honduras y Nicaragua-. El presente artículo analiza la región fronteriza del Golfo de Fonseca a través del concepto de interfaz. No se limita al registro de la confrontación diplomática entre los Estados colindantes, sino que explora la dimensión de contacto que ofrece la interfaz. El caso del Golfo de Fonseca nos parece de gran interés porque pone en evidencia las múltiples dimensiones de una interfaz compleja, a la vez multiescalar y multidimensional, como las siguientes: una interfazpolítica en construcción, una interfaz apropiada por la sociedad civil y, finalmente, una interfaz hoy ampliada que se extiende desde una dimensión transfronteriza a una dimensión trinacional.

Palabras clave: cooperación transfronteriza, actores locales, sociedad civil, actores transnacionales, ONG, ayuda internacional.

Abstract: The Gulf of Fonseca region is better known in Central America for the disputes in its boundaries that have opposed secularly the three littoral States of El Salvador, Honduras and Nicaragua. This article aims at understanding the border region of the Gulf of Fonseca through the interface concept, not limited to the mere entry of diplomatic confrontation between adjacent States, but to explore deeper the contact dimension that provides the interface. The case of the gulf of Fonseca is of great importance because it leads to propose the multiple dimensions of a complex interface, at the same time a multiscale and multidimensional interface: a politics interface in construction, an interface taken by civil society, and lastly an interface extended nowadays from a cross-border dimension to transnational dimension.

Keywords: cross-border cooperation, local stakeholders, civil society, transnational stakeholders, NGO, international founding.

Lucile Medina, doctora en Geografía por la Universidad París 10-Nanterre, docente-investigadora titular en la Universidad Paul Valéry Montpellier 3, Francia. Temas de especialización: dinámicas transfronterizas, interacciones sociales y económicas, construcción de procesos de desarrollo transfronterizos. Correo electrónico: lucile.medina@univ-montp3.fr.
Enviado a dictamen: 21 de febrero de 2013. Aprobación: 20 de abril de 2013.

Revisiones: 1 . 


\section{Introducción}

$\amalg$ n el claro contexto del fortalecimiento de las lógicas transnacionales que atraviesan el Istmo centroamericano, el Golfo de Fonseca se presenta hoy en día como una zona de interés fundamental y un ejemplo ilustrativo de las reconfiguraciones territoriales en la región centroamericana. La construcción de la nueva terminal portuaria de Cutuco en la ribera salvadoreña y el proyecto - con segmentos ya construidos - de un corredor transoceánico hacia la costa del Caribe en el marco del Proyecto Mesoamérica han provocado que la atención sobre la zona se renueve. Sin embargo, la dimensión trinacional de la región del Golfo de Fonseca confiere a la zona una complejidad adicional dada la situación secular de contacto tenso entre los Estados ribereños: El Salvador, Honduras y Nicaragua. Tradicionalmente, la literatura se ha enfocado en los diferendos de soberanía y en las protestas sobre las delimitaciones fronterizas de la región. El presente artículo pretende cambiar de enfoque y propone analizar la región fronteriza del Golfo de Fonseca a través del concepto de interfaz. El término interfaz procede de la palabra inglesa interface. Es heredado a partir de la biología y lo retomó ampliamente la informática. Remite a un dispositivo de contacto entre dos sistemas, que para las ciencias humanas y sociales pueden ser de naturaleza humana o territorial. En el campo de la geografía en particular, el uso de este concepto se ha difundido desde hace una década en la literatura en lengua inglesa o francesa, tal como lo señalan diversos artículos (Debrie y De Guio, 2004; Ó. Dochartaigh, 2007; Groupe de Recherches Interfaces, 2008; Nelles y Walther, 2011; Lerner y Eakin, 2011), revistas (Mosella, 2006), monografías (Lampin-Maillet et al., 2010; Lecoquierre, 2010; Boutoux d'Hautefeuille, 2012), así como coloquios y seminarios. En estos documentos se considera el concepto de interfaz como un instrumento de análisis operativo para abordar los espacios clave de interrelaciones - así como las interfaces portuarias, transfronterizas o periurbanas-. Retomando las reflexiones del Grupo de Investigaciones Interfaz (Groupe de Recherches Interfaces, 2008), este concepto contribuye a "interesarse menos por las limitaciones de las discontinuidades que por las interfaces entre sistemas espaciales o sociales contiguos y/o interrelacionados." El aporte es notable, "en un mundo muy organizado por las redes [...], la entrada por las interfaces y las interrelaciones que facilitan, tienden a aclarar la comprensión de la organización del espacio y de sus dinámicas actuales." Apropiarse del concepto de interfaz en el caso estudiado significa escoger ir más allá del registro limitado de la confrontación diplomática entre Estados colindantes para explorar la dimensión de contacto que ofrece la interfaz, la cual destacan los estudios actuales sobre cooperación transfronteriza (Altmann y Beirute, 2011). El caso del Golfo de Fonseca nos parece de gran interés porque nos conduce a revisar una dimensión de interfaz compleja, a la vez multiescalar y multidimensional. Primero, mostraremos la emergencia de una interfaz política que corresponde a un cambio de discurso político, con un acercamiento diplomático que busca superar los problemas de límites. Posteriormente analizaremos la realidad de la cooperación transfronteriza, poniendo en evidencia el dinamismo de los actores locales, alentado por las movilizaciones de la sociedad civil frente a la ausencia de cooperación institucional. Para finalizar, insistiremos en la fuerte presencia de nuevos actores, los donantes internacionales, que amplían la dimensión transfronteriza de la interfaz y la dirigen a un nivel claramente transnacional. ${ }^{1}$

\section{Emergencia de una interfaz política}

\section{Una región transfronteriza de contacto secularmente tenso}

El Golfo de Fonseca es una bahía amplia, de casi 2000 kilómetros cuadrados, que se abre hacia el Pacífico y cuyo territorio se extiende en tres Estados costeros de oeste a este: El Salvador, Honduras y Nicaragua. Las tensiones entre los tres actores estatales son históricas y se relacionan con asuntos de soberanía. Cada Estado nacional de la región del Golfo presenta un interés estratégico y económico importante, sobre todo El Salvador, país que tiene instalado en el Golfo de 
Fonseca su mayor puerto - La Unión, y su extensión reciente, Cutuco-, y Honduras, que posee la mayor extensión de costa en el golfo, la cual constituye su única apertura hacia el Pacífico. Para Nicaragua reviste menor importancia, pero también para este país es una zona de intercambios relativamente intensos con los dos Estados vecinos. Para ilustrar la encrucijada entre estos intereses, cabe mencionar que esta bahía compartida es el objeto del más antiguo litigio sobre las fronteras marítimas del Istmo centroamericano (Granados Chaverri, 2000).

Nuestra contribución no pretende profundizar en el aspecto jurídico de la disputa, pero es imprescindible tener presente este trasfondo político para apreciar mejor el juego actual de los actores. La abundante literatura que se ha interesado por el Golfo de Fonseca se enfoca en el tema jurídico, por lo general desde una visión partidista. Entre las numerosas referencias, destaca la síntesis del hondureño Roberto Herrera Cáceres, Estatuto jurídico de la Bahía de Fonseca y régimen de sus aguas adyacentes (1974). Más recientemente también se encuentra el trabajo de doctorado de derecho público de Gustavo Jalkh presentado en Francia, Lestatutjuridiquedu Golfe de Fonseca à la lumière de l'arrêt de la Cour Internationale de Justice dans l'affaire Honduras-El Salvador (1995). ${ }^{2}$

Existen discrepancias marítimas muy antiguas relacionadas con la soberanía de las aguas de la bahía y de los espacios marítimos más allá de la línea fronteriza. La cuestión de la delimitación de las soberanías sobre las aguas del golfo surge al final del siglo XIX. La primera tentativa de delimitación de las aguas del golfo data de 1880 y casi 40 años después, en 1917, se firma la primera sentencia de la Corte Centroamericana de Justicia sobre este tema. A lo largo del siglo XX no se resolvieron las reivindicaciones en pugna entre El Salvador y Honduras -no solamente en el golfo, sino también en segmentos de la frontera terrestre-. Ello llevó a una nueva solicitud de arbitraje, esta vez ante la Corte Internacional de Justicia de La Haya, cuya sentencia se emitió en 1992. Dicha resolución confirma el estatus de bahía histórica y de mar cerrado del Golfo de Fonseca. Asimismo, cada Estado tiene la soberanía exclusiva en una franja de tres millas a partir de la costa, mientras que las aguas de la porción central pertenecen conjuntamente a los tres Estados. Sin embargo, cabe destacar que la decisión de la Corte no puso término a algunos puntos controversiales y la partición de las aguas del golfo todavía permanece como objeto de tensiones.

Esta breve reseña muestra cómo la dimensión política trinacional contribuye a una colindancia conflictiva. Tal como lo subrayaron varios autores (Nelken-Terner, 1997; Granados, 2000), se debe tomar en cuenta la dificultad suplementaria para demarcar precisamente las fronteras marítimas, y las aguas del golfo no son una excepción en este ámbito. A raíz de la delimitación de las soberanías fijada por la Corte Internacional de La Haya en 1992, las operaciones de demarcación de las aguas del golfo sólo han sido una iniciativa fracasada. La República de Taiwán había otorgado sin embargo una ayuda financiera para llevar a cabo las operaciones de posicionamiento de boyas luminosas en seis puntos importantes a lo largo de la línea de 64 kilómetros definida en 1992 entre Honduras y El Salvador. Estas boyas hubieran contribuido a materializar la línea fronteriza y así aclarar los límites de las soberanías. Sólo se inauguró una boya maestra en 1998, que fue seccionada varias veces y que finalmente se perdió derivando en las aguas del golfo. Nunca se estableció con claridad la responsabilidad de estos sabotajes. Los pescadores hondureños atraen sospechas, siendo acusados por los nicaragüenses de buscar aprovecharse de la imprecisión en las soberanías de las aguas del golfo, resultado de la ausencia de demarcación, para extender su zona de pesca. Estas acusaciones son un ejemplo de cómo el punto de discordia en la actualidad se centra en el tema de la pesca. La falta de una clara delimitación en el golfo también provoca frecuentes roces entre pescadores de El Salvador y Honduras y militares de Nicaragua, y además altercados entre fuerzas armadas de los diferentes países. Por ello, en abril de 2012 surgió un proyecto de instalación de otras 20 boyas en el Pacífico, con un costo de 500000 dólares, para impedir futuros incidentes en el golfo.

La intervención de un Estado asiático en la disputa es sintomática. No se trató de una intervención aislada, pues en noviembre de 2012 Taiwán donó también 150000 
dólares para la construcción de un obelisco de 15 metros en la confluencia de las fronteras entre El Salvador, Guatemala y Honduras como símbolo de la integración regional. Dicha presencia no sorprende si se considera la creciente presencia de los países del sureste de Asia en América Latina, dado que los fondos destinados para la ayuda al desarrollo de estos últimos vienen a la par de intereses económicos. Los intereses asiáticos son actualmente muy perceptibles en la región y la competencia que libran entre sí las potencias asiáticas para penetrar los mercados latinoamericanos está en crecimiento.

Esta rápida exposición muestra que las diferencias relacionadas con los límites, la circulación marítima y el uso de las aguas son factores todavía presentes y se observan en las relaciones estatales. No obstante, el nivel de tensión tiende a reducirse por el proceso de acercamiento entre los Estados.

\section{Un cambio de discurso: del enfrentamiento a la apertura de una interfaz de diálogo}

Desde hace dos décadas se puede observar por lo menos un cambio de discurso por parte de los tres Estados, el cual manifiesta una voluntad marcada de acercamiento y de diálogo.

El acuerdo más global y que tuvo más relieve político fue la firma de la Declaración de Managua el 4 de octubre de 2007 por parte de los presidentes de los tres países: Manuel Zelaya, de Honduras; Elías Saca, de El Salvador; y Daniel Ortega, de Nicaragua. Firmado en presencia del secretario general de la Organización de Estados Americanos (OEA) y de los representantes del Sistema de Integración Centroamericano (SICA) y del Parlamento Centroamericano (PARLACEN), el acuerdo declara el Golfo de Fonseca "zona de paz, de desarrollo sostenible y de seguridad". Los discursos pronunciados ese día constituyen para el mundo un ejemplo de madurez política "con el objeto de profundizar y ampliar las relaciones de hermandad, entendimiento y cooperación.” A través de un diálogo presentado como "franco y constructivo", los tres países se comprometieron a llevar a cabo "programas y proyectos conjuntos que reduzcan la vulnerabilidad de los ecosistemas y poblaciones" y el "desarrollo de proyectos y empresas binacionales o trinacionales." En la exhibición mediática de lo que se presentó como una "nueva era de colaboración", nadie pareció acordarse de que una declaración trinacional similar, con los mismos objetivos de acercamiento, se había firmado en febrero de $2002^{3}$ para olvidarse poco después.

El acuerdo de 2007 preveía instalar una Comisión tripartita cuya misión sería presentar proyectos para sostener el desarrollo regional y reducir la vulnerabilidad de los ecosistemas. Tenía también como objetivo regular los conflictos entre los guardacostas y los pescadores acusados de pesca ilegal por cada uno de los países. El acuerdo contemplaba otros proyectos de acercamiento, como la creación de una Universidad del Mar o la reapertura de una línea de ferry para comunicar la costa salvadoreña con la nicaragüense - cerrada ésta durante la época sandinista-

En el contexto propicio del encuentro de los presidentes en 2007, cabe notar, sin embargo, algunas discordancias. La propuesta reiterada del presidente salvadoreño Elías Saca de que se declarara un régimen de condominio en el golfo era una de las más delicadas. Se sabe que Honduras se oponía a esta interpretación, y volver sobre esta cuestión fronteriza, todavía polémica, es un detalle revelador de la fragilidad de las declaraciones de acercamiento entre los protagonistas que no terminan de hacer borrón y cuenta nueva del pasado. La complicación más importante a la cual los proyectos tuvieron que hacer frente se produjo a partir de 2009 con motivo de la crisis política que vivió Honduras. En una declaración de agosto de 2010, ${ }^{4}$ Daniel Ortega buscaba reanudar las relaciones suspendidas entre Nicaragua y Honduras y el trabajo de comisión trinacional, entonces inactiva. Posteriormente, en el año 2012 se reunieron varias veces los tres presidentes en ejercicio: Daniel Ortega, de Nicaragua, Porfirio Lobo, de Honduras, y Mauricio Funes, de El Salvador. En esa reunión, nuevamente manifestaron su interés por reactivar la Declaración de Managua y trabajar en la formulación de un Plan de Desarrollo Sostenible para el Golfo de Fonseca; a la par reafirmaron su posición de 
apaciguamiento frente al escenario tenso otra vez en las aguas del Golfo. Tras cuatro incidentes en el transcurso de ese año entre las fuerzas navales de Honduras, El Salvador y Nicaragua, ${ }^{5}$ los tres presidentes anunciaron a principios de diciembre de 2012 la creación de una comisión trinacional que se encargaría de trabajar en las delimitaciones fronterizas en el Golfo de Fonseca. ${ }^{6}$

Sin embargo, la compleja cuestión del reparto de soberanía de las aguas del golfo no deja de reivindicarse de una manera abierta y franca en la mesa de discusiones. En tales condiciones, a pesar de que los discursos pretendían hacer creer lo contrario, se constató la dificultad que tenían los tres Estados para avanzar en el tema del desarrollo regional y en la elaboración de una estrategia común. La cooperación transfronteriza institucional, liderada por los actores estatales, está inevitablemente comprometida. Sin embargo, es en la esfera de la sociedad civil donde emergen iniciativas, las cuales manifiestan el dinamismo a escala local y el deseo de acercamiento más allá de las fronteras nacionales.

Además, este cambio de registro, de la confrontación al acercamiento, debe analizarse a la luz de los retos económicos actuales. La nueva imagen de estabilidad política dada a la región del golfo y el alarde de relaciones cordiales entre los tres Estados costeros parece participar de una voluntad de tranquilizar y atraer a potenciales inversionistas.

Además de las inspecciones de barcos vinculadas al problema de la imprecisión en la demarcación fronteriza en el golfo, la atención reciente se ha enfocado en la construcción de un nuevo puerto en la costa salvadoreña, Cutuco, extensión del puerto ya existente de La Unión, con vocación de plataforma de redistribución regional que puede acoger barcos de tamaño post-Panamá. El proyecto de ampliación debería completarse con una zona de empresas, entre las cuales se encontrarían dos plantas eléctricas. Es precisamente el impacto ambiental el que aparece como tema más discutido y el que ha movilizado a los oponentes al proyecto a lo largo de su construcción.

El contexto político, así como el económico, no son ajenos a la emergencia de la movilización de los actores locales, la cual surgió en un primer momento como oposición local circunscrita a los marcos nacionales, para desbordar hoy las fronteras y adquirir una dimensión transfronteriza. En el doble marco de la ausencia in fine de cooperación institucional a escala estatal y de la emergencia de fuertes preocupaciones en los ámbitos económico y ambiental, es donde se observan acercamientos locales y la construcción de puentes transfronterizos de otra índole.

\section{Una interfaz entre cooperación institucional e iniciativas de la sociedad civil}

\section{La deficiencia de la cooperación institucional}

La región que bordea el Golfo de Fonseca presenta, a pesar de las diferencias diplomáticas que agitan sus aguas, fronteras permeables animadas por los movimientos de la población y por los intercambios diversos, tanto de carácter formal como informal. Las poblaciones fronterizas están tradicionalmente en contacto y mantienen vínculos constantes. Ésta es una de las razones por las que el Golfo de Fonseca aparecía en 1995 entre los 15 lugares identificados por las instancias de integración regional como prioritarios para impulsar acuerdos de cooperación transfronteriza. La firma por parte de los vicepresidentes del Istmo centroamericano del documento que presentaban estas 15 regiones transfronterizas prioritarias, el "Acuerdo sobre el desarrollo sostenible fronterizo en Centroamérica", debe situarse en el contexto de la reactivación del proceso de integración regional a partir de 1990. Este acuerdo constituía un paso adelante en la dinámica de cooperación transfronteriza, dado que consideraba ésta de manera explícita, y por primera vez, como un "instrumento de integración". En toda la región centroamericana es muy claro el impulso dado por las instancias supranacionales a los proyectos de acercamiento y de cooperación, aunque es evidente que no tienen los medios de acción suficientes para acompañar estos programas. Debido a su dependencia de los Estados nacionales, las firmas y la implementación de los acuerdos de cooperación transfronteriza ofrecen 
en las fronteras de Centroamérica, casi 20 años después, una amplia variedad de situaciones. Ello refleja esencialmente la diversidad de las relaciones diplomáticas que mantienen los vecinos.

Se debe subrayar, además, el desfase general existente entre lo que atañe al discurso -interesante por sí mismo - y el cumplimiento efectivo de las intenciones mostradas. Nuestro propósito no es debatir sobre las acciones efectivas de los programas, cuyo retraso se podría atribuir en parte a la escasez de financiamientos nacionales, sino centrarnos en el nivel de tensión política entre los países y en la falta de voluntad de superarla. Consideramos necesario aclarar que analizamos el discurso y no el avance efectivo de los programas de cooperación. Precisamente no existe ningún acuerdo de cooperación alrededor de la triple frontera del golfo. Esa ausencia de cooperación institucional se explica en la región por dos causas fundamentales: por un lado, se debe a los conflictos políticos internos de cada Estado, que han absorbido la atención de sus dirigentes y, por el otro, al persistente desacuerdo relativo al reparto de las aguas del golfo. Sin embargo, el contexto político ha evolucionado durante estos últimos años y está marcado por dos acontecimientos que actúan en sentidos opuestos: el primero es propicio a la cooperación, mientras que el segundo la ha afectado. El cambio de discurso político es evidente desde 2007, fecha a partir de la cual se manifestó una voluntad de acercamiento. Pero el golpe de Estado en Honduras en 2009, condenado por la comunidad internacional, puso un alto a las discusiones, ya poco consistentes de por sí, y volvió a sumir a la región en los errores del pasado. Finalmente, a pesar del principio de acuerdo de 2007, nada se ha avanzado desde entonces, ni siquiera se estableció la comisión trinacional cuya creación se había anunciado.

Así pues, a pesar de las declaraciones diplomáticas, no es del lado de los Estados donde se pueden observar avances en materia de cooperación transfronteriza en el Golfo de Fonseca. En cambio, se afirma que la cooperación se estableció a través de un marco diferente y novedoso, impulsada en el nivel local por los actores municipales y de la sociedad civil.

\section{Las asociaciones transfronterizas \\ conformadas por actores locales}

Existen tres iniciativas de este tipo en la actualidad: dos que se ubican en el campo asociativo y una en el campo político local. Las tres vienen en cierta manera a llenar el vacío institucional y a dar cierta realidad global a la región del golfo.

La primera de ellas es ACTRIGOLFO. Se trata de una asociación u organización no gubernamental (ONG) ${ }^{7}$ trinacional, creada en 1994. Conformada al principio por seis asociaciones —dos salvadoreñas, una hondureña y tres nicaragüenses ${ }^{8}$, cuenta hoy con 16 miembros. Inició como asociación sin fines de lucro bajo el impulso de la Comisión Centroamericana de Ambiente y Desarrollo (CCAD) para fortalecer la integración regional. En su fundación estuvieron presentes representantes de los Estados, además de patrocinadores como las ONG Greenpeace y Unión Internacional para la Conservación de la Naturaleza (UICN).

Otra de las asociaciones, PescaGolfFo, también trinacional, está conformada por pescadores del Golfo de Fonseca. Nació en 2007 por iniciativa de las asociaciones locales de pescadores artesanales de las tres riberas. Varias asociaciones miembros de PescaGolFo pertenecen también a ACTRIGOLFO. Desde 2007, esta asociación se ha movilizado sobre todo para abordar el problema recurrente de las zonas de pesca - los pescadores ven sus barcos detenidos y son multados-, pero también en contra de la construcción del puerto de Cutuco, que ha requerido dragados muy importantes y con desastrosos efectos para la pesca. PescaGolFo tiene una acción sectorial y, por consiguiente, no tiene la amplitud de ACTRIGOLFO ni su dinámica de acción.

Finalmente, MUGOLFO constituye la asociación de las municipalidades del Golfo de Fonseca. Su acta de conformación la firmaron el 14 de diciembre de 2011 los 22 alcaldes comprometidos. Esa iniciativa da luz a una mancomunidad trinacional con base en el reagrupamiento de las siete mancomunidades que existen alrededor del golfo - una en El Salvador, cuatro en Honduras y dos en Nicaragua-. Su objetivo consiste 
en contribuir al mejoramiento de las condiciones y de la calidad de vida de los pobladores del golfo. ${ }^{9}$ El proceso de diálogo emprendido en 2008 fue impulsado por el SICA y el programa de cooperación italiano Fronteras Abiertas ${ }^{10}$ para intentar enfrentar las diferencias jurídicas de competencias entre las mancomunidades de los tres países. No se trata sin embargo de una experiencia pionera en Centroamérica porque ya existe una mancomunidad trinacional en la frontera entre Guatemala, El Salvador y Honduras, organización que apadrinó, junto con otras instituciones, la creación de MUGOLFO. Se puede también citar la conformación en 2001 de una asociación similar en la triple frontera entre Bolivia, Perú y Chile (Rouvière, 2009), aunque en este caso el acercamiento se fundamentó en bases étnicas - comunidades aimaras-, lo cual no ocurre en Centroamérica.

Aunque los cimientos de estas iniciativas recientes no son siempre estables, llaman sin embargo la atención. Por consiguiente, es ineludible cuestionar el sentido que debe darse a esta dinámica de estructuración asociativa en la escala transfronteriza.

La reivindicación de una gobernanza compartida es el elemento clave que ha de comprenderse. Fue posible gracias a la estabilización política y a la transición democrática porque ambos factores han permitido la apertura de un proceso de descentralización y, paralelamente, de un espacio de expresión para la sociedad civil. Cabe mencionar que este proceso ha sido tardío en Centroamérica, donde varios países han sufrido conflictos internos muy violentos hasta los años noventa del siglo pasado. Si Honduras empezó su transición democrática en 1982, las elecciones libres que pusieron fin a la revolución sandinista datan de 1990 y los Acuerdos de Paz en El Salvador solamente de 1992. La conformación de ACTRIGOLFO en 1994, es decir, dos años después, se inscribe directamente en este nuevo contexto. En tal sentido, se observa cómo los actores locales reivindican su participación en una gobernanza compartida en varias escalas - multi level governance- ACTRIGOLFO se posiciona como "facilitador de la sociedad civil", referencia hoy ineludible para la definición de todos los proyectos de desarrollo, según el principio que establece la legitimidad de los programas de desarrollo a partir de la participación de los pobladores en su concepción. Las mancomunidades igualmente insisten, a través de la federación transfronteriza MUGOLFO, en la necesaria participación de los niveles políticos locales en los proyectos de desarrollo.

Las estructuras transfronterizas estudiadas se ubican en un doble registro: la denuncia debido a la ausencia de información y de consulta ciudadana- ${ }^{11}$ y la negociación. En este último tema, los actores involucrados en el movimiento asociativo transfronterizo militan para su participación activa en la definición de los proyectos. Los actores locales reivindican su capacidad de evaluación y se niegan a limitar su rol al de ejecutantes de apoyo a la implementación de los programas; dicho de otro modo, se niegan a ser simples suministradores de servicios. Así, una de las prioridades de ACTRIGOLFO consiste en tomar posición como agente interlocutor ante las instancias gubernamentales y supranacionales. En 2007, tras la firma de la Declaración de Managua, esta asociación solicitó a los cancilleres de los tres Estados su integración en calidad de observadora en la comisión trinacional que se proyectaba iniciar - la cancillería hondureña dio una respuesta positiva-. PescaGolFo hizo lo mismo en su búsqueda por tomar parte activa en las discusiones. Ello remite a un enfoque "institucional", comprendido éste como: "buscando ante todo influenciar directamente la gobernanza regional, principalmente a través de la participación, como observador, consultante y a veces suministrador de servicios, en los proyectos ejecutados por los gobiernos" (Buclet, 2006, traducción de la autora). En esta búsqueda, los actores locales se enfrentan a la pasividad y a la inercia de los gobiernos, aunque estos últimos aceptan en apariencia jugar el juego para sumarse a los preceptos globales de "buena gobernanza".

Estas asociaciones transfronterizas contribuyen sin embargo a estimular la relación de dependencia que une estas regiones periféricas y atrasadas con las capitales, actitud que tiende a cambiar la lógica de exclusión el alcalde de La Unión se refiere a las poblaciones del 
Golfo como "huérfanas" - hacia una nueva lógica de inclusión. Demuestran que, a escala local, los actores no permanecen pasivos hoy en día frente a los proyectos impuestos desde arriba - top down - o que tardan en realizarse. Intentan impulsar una dinámica bottom up con la idea planteada de una "cogestión del Golfo". ${ }^{2}$ La cogestión que se reivindica puede interpretarse en las zonas fronterizas a partir de dos dimensiones: en un plano horizontal, entre actores locales de las tres riberas del golfo, y desde un punto de vista vertical, entre actores locales y estatales. Esto remite a un enfoque más militante, mostrando que las asociaciones y ONG que actúan en el ámbito del desarrollo pueden ahora combinar fácilmente los enfoques militante e institucional.

Este nuevo juego de actores desplaza la geometría de los conflictos. Los antagonismos tradicionales oponían a los tres Estados ribereños del Golfo de Fonseca por diferencias de soberanía y de explotación de los recursos. Hoy en día, las tensiones interestatales han visto bajar su intensidad y la oposición parece cristalizarse más verticalmente, entre el nivel local y los gobiernos centrales, en relación con la aspiración de los primeros a captar la atención de los segundos.

Sin embargo, no hay que pasar por alto las relaciones entre actores locales, las cuales deben cuestionarse. Según nuestro conocimiento, parecen más bien buenas. La estructura abierta de foro que desea conservar ACTRIGOLFO es interesante. Además de las asociaciones que participan como miembros, también se invita a las reuniones a las municipalidades y las mancomunidades de la zona, lo cual constituye una manera de establecer un espacio de diálogo entre todos los actores locales, tanto civiles como políticos, para construir reivindicaciones comunes. Sin caer en el error de reducir la sociedad civil a un cuerpo virtuoso y homogéneo frente al Estado, se debe reconocer que, en los casos estudiados, por el momento se observa en ella una voluntad de mantenerse en una línea unida. Las razones son claras: se respaldan en un conjunto de ideas cercanas y compartidas en cuanto a los retos del desarrollo regional, así como en un interés por promover estas ideas de forma más eficaz entre los actores nacionales, y también entre los actores transnacionales considerados como ineludibles.

\section{Una interfaz con una dimensión transfronteriza y transnacional}

El movimiento de alianzas transfronterizas, testigo de una mutación en las escalas de acción política

Edith Antal (2005), en el marco de un análisis comparado de dos regiones en las fronteras norte y sur de los Estados Unidos, hizo notar un desplazamiento en las escalas de acción política que identifica el paso de un eje local-nacional hacia un eje regional-internacional. El caso del Golfo de Fonseca evidencia el mismo fenómeno y nos lleva a pensar los territorios transfronterizos como particularmente representativos de esta mutación, fenómeno observable también de manera más amplia. Las alianzas estudiadas que se construyen en la escala transfronteriza manifiestan esta mutación en las escalas de acción, mientras que se observa una interacción progresiva entre la escala regional — transfronteriza-y la transnacional.

Este cambio viene acompañado por una complejidad creciente de los juegos de los actores. Actualmente, se hace imperiosa la necesidad de ubicarse y navegar en la nebulosa de los actores. Va en este sentido la publicación de un documento titulado Mapa de actores en desarrollo humano y medio ambiente en el Golfo de Fonseca (2010), realizado por la CCAD y destinado a los actores del desarrollo.

\section{Desde lo local a lo transfronterizo: la aprehensión de un territorio compartido}

La conformación de alianzas en la escala transfronteriza marca el paso de una dimensión fronteriza a una lógica transfronteriza como radio de acción y como escala de reflexión territorial. ACTRIGOLFO nació precisamente de la "necesidad de considerar el Golfo como una entidad, como una unidad, un territorio."13 Así, el lema de la asociación es "tres países, un solo Golfo." La defensa de un medioambiente compartido constituye el 
motor primordial. El lema de MUGOLFOes, por su parte, muy similar: "El Golfo no es solamente del Salvador, de Honduras o de Nicaragua, lo compartimos." La firma de la Declaración de Managua en 2007 parece haber contribuido a que todos los actores "vean el Golfo."14 Las representaciones van cambiando y la realidad de un territorio transfronterizo parece dibujarse poco a poco, bajo el efecto eficiente del discurso, así como en la realidad de las prácticas que dan sentido a esa nueva entidad. Lo que está en juego es la inscripción de lo transfronterizo como una nueva escala de la acción territorial.

Sin embargo, los puntos frágiles de las alianzas estudiadas son numerosos. La búsqueda de fuentes de financiamiento es el primer reto, porque sin recursos ninguna acción resulta posible. Existen muchas limitaciones financieras que condicionan el funcionamiento de las asociaciones, incluso para organizar reuniones.

Los actores identifican también las diferencias de legislación entre los tres Estados como un factor limitante para una acción integrada. El sector ambiental se cita con frecuencia; por ejemplo, las normas y categorías de las áreas protegidas difieren ampliamente y obstaculizan el trabajo común.

El riesgo que la inestabilidad política genera no es la menor espada de Damocles que abruma los proyectos transfronterizos. La agitación política que afecta a Honduras desde 2009 ha tenido consecuencias negativas en los proyectos de desarrollo de la región, lo que demuestra que la estabilidad política es el factor esencial de éxito gracias al clima sereno y propicio que ofrece a la cooperación. El avance de los programas en el Golfo de Fonseca ha padecido con los bloqueos en los puestos fronterizos de Honduras, y varias fuentes de financiamiento nacionales e internacionales se mostraron reticentes a ayudar indirectamente a un gobierno surgido de un golpe de Estado.

Finalmente, el mantenimiento de la unidad más allá de las fronteras es el último obstáculo por superar. Para el gerente de ACTRIGOLFO, el reto más grande de la asociación es sencillamente "mantenerse, mantenerse unidos." Ello pasa por la necesidad de reunirse. Para ello, las herramientas TIC, el teléfono, el correo electrónico o la comunicación vía Skype, si bien ayudan mucho, no substituyen totalmente los encuentros físicos. Ahora bien, el ritmo de los encuentros se apoya en el dinamismo de sus miembros y no todos se implican de igual forma. Tres asociaciones, una en cada país, son las más activas, los "referentes", y dan el impulso: CODECA de El Salvador, COODEFFAGOLF de Honduras y LIDER de Nicaragua. ${ }^{15}$ La cohesión de ACTRIGOLFO se debe en gran parte también a la contratación en 2009 de un gerente encargado de dar vida a la estructura transfronteriza, asegurando la coordinación financiera y administrativa de la asociación, "sobre todo manteniendo la red, que nadie se sienta olvidado, que todos estén informados de los proyectos, de las oportunidades." ${ }^{\text {6 }}$

\section{De lo transfronterizo a lo transnacional: la afirmación de la cooperación internacional en el seno de las dinámicas transfronterizas}

La elaboración de varios programas diferentes a partir de la década de los noventa del siglo pasado, si bien se tradujo en una multiplicación de las acciones y demostró falta de coherencia - y finalmente una falta de legibilidad-, es también un síntoma de la omnipresencia, y aún del predominio actual, de nuevos actores externos en los proyectos de desarrollo fronterizos. Esa novedad ha sido ya subrayada y corresponde a una evolución nítida de la gobernanza de los espacios transfronterizos en América Latina que ve cómo aumenta la presencia a la vez de los actores locales y de los actores transnacionales (Rouvière, 2009).

Entrelosagentesexteriores presentescomprometidos en la creación y el funcionamiento de las asociaciones u ONG y de los programas transfronterizos de la región del Golfo de Fonseca, destacan:

- Los organismos supranacionales de integración —el SICA, a través la CCAD-.

- Las organizaciones intergubernamentales tales como los bancos regionales de integración - por ejemplo el Banco Interamericano de Desarrollo- 
y los organismos de ayuda multi o bilaterales. Los cuatro principales identificados por la CCAD en la región del Golfo (2010) son la cooperación española Programa BID GEF-, ${ }^{17}$ la cooperación estadounidense, la cooperación japonesa, la cooperación austriaca y la cooperación europea. Pero están también presentes la cooperación alemana, la cooperación italiana - programa Fronteras Abiertas - y la cooperación danesa - programa PROGOLFO-, ${ }^{18}$ organismos que financian diferentes programas.

- Las organizaciones no gubernamentales internacionales tienen más o menos un esquema de intervención similar: The Nature Conservancy (TNC) o la Unión Internacional para la Conservación de la Naturaleza (UICN) son unas de las más grandes, pero también Amigos de la Tierra - ONG española - posee sedes locales y acompaña desde el principio la existencia de ACTRIGOLFO, así como la ONG portuguesa OIKOS.

Esta larga lista nos muestra varias cosas. Primero, en lo que concierne a la orientación de la mayoría de los programas de cooperación financiados, el tema socioambiental aparece como un elemento clave, reflejando un movimiento inscrito en la filosofía de la conferencia de las Naciones Unidas de Estocolmo de 1972 bajo el lema "tenemos sólo una tierra." La invocación de la naturaleza justifica e incita a la superación funcional de las divisiones fronterizas artificiales (Fourny, 2005). Cabe notar que en muchos programas transfronterizos la preservación del medioambiente se considera a menudo como una forma de construir fácilmente algo en común y superar así las divisiones fronterizas. La presencia de los Ministerios de Ambiente en la mayoría de los programas estudiados tiende a reducir a retos ambientales las problemáticas que en estos territorios están muy lejos de poder reducirse a esta característica.

Por otra parte, la presencia de estos múltiples actores indica un paulatino proceso de sustitución de los actores estatales, los cuales por falta de voluntad y capacidad financiera no impulsan los proyectos, aunque desean mantenerse presentes.

De ahora en adelante, el impulso de la cooperación internacional es esencial. Se expresa simultáneamente a través de un apoyo al peritaje y a la logística y de un apoyo financiero. Este respaldo está destinado también a las estructuras locales, ONG o mancomunidades. Así, la cooperación española (AECID) respalda a ASIGOLFO, en la ribera salvadoreña, desde su creación. La confluencia observada entre las aspiraciones locales y el respaldo internaciona ${ }^{19}{ }^{19}$ remite al análisis que William Robinson (2003) hizo de las sociedades civiles en Centroamérica, mostrando que se vuelven "transnacionalizadas" porque evolucionan fuertemente en vínculo con los actores globales.

\section{Lo transfronterizo es bankable $e^{20}$ ante lo transnacional}

De hecho, el sello "transfronterizo" se convirtió en un medio de captación de recursos internacionales. La búsqueda de fuentes de financiamiento se inscribe como la preocupación mayor entre el conjunto de actores políticos y asociativos locales: así, "captar" fue la palabra más pronunciada en una de las entrevistas. Robinson (2003) lo relaciona con un cambio en las estrategias de alianzas desde los años noventa y con el fortalecimiento de las ONG. Para él, se trata, por parte de las ONG, de una redirección hacia la sociedad civil de la ayuda antes dada a los Estados. En el mismo sentido, se puede citar un artículo de Planche y Lavigne (2005) dedicado a la posición de la Unión Europea ante las sociedades civiles del sur en las acciones de cooperación. Los autores afirman que "la problemática del fortalecimiento de las organizaciones de la sociedad civil forma parte integral de los discursos y del repertorio de acción del conjunto de los actores del desarrollo," y que ahora aparece omnipresente en las acciones tanto de las ONG como del Banco Mundial o de los donantes internacionales.

La construcción de alianzas transfronterizas se fundamenta, además de en la comprobación de problemáticas comunes, en la convicción de que el sello "transfronterizo" ofrece a los actores locales una mayor visibilidad y, por consiguiente, es el mejor 
medio para captar los financiamientos de los donantes y hacer elegibles sus programas. La transfrontericidad es bankable, así como lo expresa Laetitia Rouvière en su análisis de la asociación de municipalidades aimaras en la frontera entre Bolivia, Perú y Chile (2009). Esa estrategia pragmática surge de la experiencia adquirida por los actores locales en la manera de venderse para garantizar su supervivencia. "Carecemos de recursos propios para poder elaborar un proyecto, la idea es pues poder captar financiamientos." ${ }^{21}$

Si bien estos financiamientos permiten respaldar las iniciativas transfronterizas y desempeñan así un papel positivo, su naturaleza es sin embargo problemática porque induce a una dependencia de los donantes y pone en peligro la continuidad de las acciones y la viabilidad misma de las estructuras. El ejemplo de ACTRIGOLFO es significativo. Además del respaldo fundamental de la CCAD, la asociación se ha beneficiado también directamente de los fondos de la ONG española Amigos de la Tierra y de la cooperación alemana (DED). Otras ONG han estado, o todavía están, presentes a través de programas más puntuales: The Nature Conservancy, WWF, la UICN y Geólogos del Mundo. ACTRIGOLFO ha pasado así una primera fase activa hasta 1999, durante la cual participó en la ejecución de un programa de preservación de los ecosistemas costeros del Golfo de Fonseca impulsado por la CCAD (PROARCA/COSTAS) y financiado conjuntamente por la Agencia Estadounidense para el Desarrollo (USAID), Rainforest Alliance, WWF y TNC. El fin del programa PROARCA ha acarreado, entre 2000 y 2004, una fase de desmovilización. Desde 2004, el apoyo de la UICN ha permitido la supervivencia de ACTRIGOLFO, aunque el golpe de Estado en Honduras provocó la interrupción de la ayuda financiera y de los programas asociados por parte de la cooperación alemana y de varias ONG.22 El Plan Estratégico 2011-2015 parecía poder asegurar un financiamiento a cargo del equipo técnico Corredor del Mangle (CCAD y AECID), pero las últimas entrevistas realizadas permiten concluir que ACTRIGOLFO se encuentra de nuevo en gran parte inactivo. ${ }^{23}$

Lo que se puede añadir finalmente es que, además de la captación de recursos, el llamado a los agentes exteriores obedece también a la necesidad de atraer a la comunidad internacional para intentar adquirir peso ante los gobiernos nacionales. Estos últimos han venido descargándose de su función de garantes del estado de bienestar —en parte por falta de recursos-y dejan hoy a los actores de la cooperación internacional — agencias de desarrollo de los Estados del norte u ONG - aportar los fondos necesarios para los diagnósticos y la implementación de programas de desarrollo. No obstante, permanecen presentes y son ineludibles en cuanto al fortalecimiento de las alianzas transfronterizas. De esta manera los actores locales esperan interpelar a los gobiernos nacionales.

\section{Conclusión}

Conviene resituar el análisis propuesto en el contexto de una atención renovada en la escala mundial para las fronteras desde el final de los años ochenta, marcada por obras de referencia como Fronts et frontières de Michel Foucher (1988) o World Boundaries, que suma cinco volúmenes, dirigida por Clive Schofield (1994). Para Centroamérica en particular, la literatura se interesa desde hace 15 años en los cambios en la manera de aprehender las fronteras (Bovin, 1997; Medina, 2007) y más precisamente en la emergencia de iniciativas de cooperación transfronteriza bajo el paradigma de la integración (Girot y Granados, 1997; Matul, 2007). Lo que muestra el presente estudio, a través del caso de la región del Golfo de Fonseca, es primero la afirmación de la dimensión de interfaz de las fronteras centroamericanas, aun cuando el contexto político es determinante y se ve que las tensiones diplomáticas dificultan los acercamientos. También pone de manifiesto la afirmación de los procesos de transnacionalización en los programas de desarrollo y más precisamente en las zonas fronterizas. Faltaría cuestionar más precisamente la influencia que la presencia central de los donantes y de la ayuda internacional puede tener en el proceso de creación de programas y acciones, y en las orientaciones de éstos. Puede ser una pista para la reflexión pero, a todas las escalas, es notable la reorganización de una 
nueva geometría de las relaciones entre los actores. Si bien los Estados siguen estando presentes y son ineludibles para propiciar la apertura de las fronteras, las zonas fronterizas aparecen hoy en día como lugares privilegiados para observar las dinámicas transnacionales y muestran la fuerte conexión que se puede establecer entre las escalas transfronteriza y transnacional.

\section{Notas}

${ }^{1}$ Este trabajo se inscribió en el marco del programa francés de investigación "Dinámicas transnacionales y recomposiciones territoriales: enfoques comparativos en los sures: Mesoamérica/Asía del Sureste" (20082011), liderado por Laurent Faret, profesor de Geografía de la Universidad París-Diderot. El análisis se apoya en una recensión bibliográfica y una serie de entrevistas realizadas entre 2008 y 2012 en los tres países ribereños del Golfo de Fonseca.

${ }^{2}$ La base de datos bibliográficos del Instituto de Historia de Nicaragua y Centroamérica (IHNCA) en Managua -Universidad Centroamericana- inventarió 23 referencias en relación con el Golfo de Fonseca.

${ }^{3}$ Francisco Guillermo Flores Pérez, presidente de El Salvador; Ricardo Maduro Joest, presidente de Honduras; Enrique Bolaños Geyer, presidente de Nicaragua

${ }^{4}$ Ver El Heraldo.hn, periódico digital hondureño. Honduras, 3 de agosto de 2010.

${ }^{5}$ Ver Laprensa.hn, 2 de diciembre de 2012.

${ }^{6}$ El fallo de la Corte de La Haya de 1992 concedió tres millas náuticas a cada país, pero ordenó que el manejo de la bocana fuera definido mediante un arreglo entre Nicaragua, Honduras y El Salvador, lo cual hasta ahora no se ha logrado.

7 Usamos los términos de asociación y de ONG de manera sinónima para designar las mismas estructuras. Los actores asociativos locales que actúan en el ámbito del desarrollo se definen más comúnmente como ONG. Sin embargo, el estatuto de ONG no existe a nivel internacional. Se trata de asociaciones de solidaridad, que no son siempre internacionales ONG nacionales.
8 Para El Salvador: Asociación Coordinadora de Comunidades para el Desarrollo del Cacahuatique (CODECA) y Asociación Unionense para la Defensa del Medio Ambiente (ASUMA); para Honduras: Comité para la Defensa de la Flora y la Fauna del Golfo de Fonseca (CODDEFFAGOLF) para Nicaragua: Somos Ecologistas en Lucha por la Viday el Ambiente(SELVA), Unión Regional de Cooperativas Camaroneras de Puerto Morazán y Potosí (URCOOCAM) y Asociación Club de Jóvenes Ambientalistas (ACJA).

9 "Nos unimos para incidir de manera significativa en el desarrollo local, trabajando de forma conjunta para superar los problemas comunes que sufren los habitantes de nuestra región", Declaración de León, 26 de julio de 2011.

${ }^{10}$ Red interregional para la cooperación transfronteriza y la integración latino-americana, cuyo principal donante es la Cooperación Italiana para el Desarrollo, 〈www. fronterasabiertas.org).

11 De manera general, las personas entrevistadas denuncian el hecho de que "no se considera lo que vive o piensa la gente" y que "los proyectos no han considerado ni el tejido social ni el ambiente" extractos de entrevistas con los miembros de ACTRIGOLFO, mayo de 2010-. Para Osmar Cruz, el alcalde del FMLN del municipio de La Unión (2009-2012), lugar donde se construyó el nuevo puerto de Cutuco, este proyecto "ha sido planificado por pocos" (entrevista, mayo 2010). Este último se expresó en la prensa sobre este punto: “ Por eso, todos los ciudadanos: profesionales, obreros y campesinos nos hemos unido para luchar contra este tipo de situaciones, porque queremos participar en el desarrollo" (ver DiarioCoLatino.com, 27 de enero de 2009). La victoria de Osmar Cruz en las elecciones municipales de enero de 2009 parece que se debe mucho a su participación activa en la asociación ecologista ASUMA y a la participación en las dos marchas organizadas en la cuidad de La Unión en 2007 en contra de los proyectos de centrales eléctricas en la zona portuaria de Cutuco.

${ }^{12}$ Ver página web de ACTRIGOLFO, <actrigolfo.net〉 [10 de octubre de 2012]. 
13 Entrevista a R.M. López, gerente de ACTRIGOLFO, mayo de 2010.

${ }^{14}$ Entrevista a R. Jiménez, director de la mancomunidad ASIGOLFO, miembro de MUGOLFO, El Salvador, 2010.

${ }^{15}$ Entrevista a R.M. López, ACTRIGOLFO, Honduras, mayo de 2010; entrevista a W. Áreas, LIDER, Nicaragua, noviembre de 2008.

${ }^{16}$ Entrevista a R.M. López.

${ }^{17}$ Desde 2006, la CCAD y la AECID vienen trabajando en el Proyecto de Gestión Integrada de Ecosistemas en el Golfo de Fonseca, patrocinado también por el BID y el GEF (Global Environment Facility). La meta es asegurar sinergias y un modelo de gestión coherente y consistente con el curso de formulación participativa. En los talleres de preparación, cabe notar la participación de 18 de los 19 municipios que bordean el Golfo. Sin embargo aún no parece haber comenzado su ejecución, por la situación política de Honduras. Mas recientemente, otro proyecto fruto de la colaboración entre la CCAD y la AECID se conformó en 2008 - Proyecto Trinacional Corredor del Mangle-: tiene como objeto — muy similar a los precedentes - "contribuir al desarrollo social sostenible y a la conservación de la biodiversidad del Golfo de Fonseca mediante acciones que mejoren la calidad de vida de sus pobladores, reduzcan la pobreza y el impacto ambiental."

${ }^{18}$ En 1994 salió a la luz el programa PROGOLFO. Fue el primer Proyecto regional para la protección de los ecosistemas costeros del Golfo de Fonseca (Diagnóstico del estado de los recursos naturales, socio-económicos e institucionales de la zona costera del Golfo de Fonseca, Resumen ejecutivo, DANIDA/Gobiernos de El Salvador, Honduras y Nicaragua, 1994).

19 En el caso estudiado no se observa competencia entre las ONG locales, las ONG transnacionales y los diferentes programas de desarrollo, sino más bien una conjunción de intereses. Sin embargo, ha podido ser el caso en otros lugares, como en Colombia, donde la implementación de un programa europeo de desarrollo rural ha generado un conjunto de conflictos cuando la ONG legítima para las autoridades políticas de la zona, y que se encontraba a la iniciativa de las negociaciones con la delegación de la Comisión Europea, perdió el control sobre el programa (Santamaría y Zuñigo, 2005).

${ }^{20}$ Bankable: palabrainglesa cuyo significado es "susceptible de recaudar fondos."

${ }^{21}$ Entrevista a R. Jiménez, ASIGOLFO, mayo de 2010.

${ }^{22}$ Rimen Martínez López, gerente de ACTRIGOLFO: "Sus prioridades ya no son aquí", "Muchos de los proyectos se han retirado de la zona" (entrevista, mayo de 2010).

${ }^{23}$ Entrevistas a Leónidas Pérez, presidente, y Marianela Santamaría, gerente técnico de ACUGOLFO, Asociación Hondureña de Cuencas del Golfo de Fonseca, miembro de ACTRIGOLFO, agosto de 2012.

\section{Bibliografía}

Altmann Borbón, Josette y Tatiana Beirute Brealey (eds.) (2011), América Latina y el Caribe: cooperación transfronteriza. De territorios de división a espacios de encuentros, Buenos Aires: Teseo/FLACSO.

Antal, Edith (2005), Nuevos actores en América del Norte. Identidades culturales y políticas, México: CISAN-UNAM.

Arauz Muñoz, Jeannette y Hannia Delgado Carazco (2002), El potencial de conflicto ambiental en la cuenca del Golfo de Honduras, San José de Costa Rica: Fundación para la Paz y la Democracia/Universidad de Costa Rica.

Boudoux d'Hautefeuille, Madeleine (2012), Entre marge et interface, recompositions territoriales à la frontière francobrésilienne (Guyane/Amapá), (tesis de doctorado en Geografía), Université des Antilles et de la Guyane, Martinique.

Bovin, Philippe (coord.) (1997), Las fronteras del Istmo: fronteras y sociedades entre el sur de México y América Central, México: CEMCA.

Buclet, Benjamin (2006), "Les réseaux d'ONG et la gouvernance en Amazonie", en Autrepart, vol. 1, núm. 37, pp. 93-110.

Bustillo Lacayo, Guillermo(2002), El Golfode Fonseca:región clave para Centroamérica, Tegucigalpa: Guaymuras. 
Comisión Centroamericana de Ambiente y Desarrollo (2010), Mapa de actores en desarrollo humano y medio ambiente en el Golfo de Fonseca, s.l.: la Comisión.

D’Ans, André-Marcel (1997), Le Honduras, difficile émergence d'une nation, d'un Etat, París: Karthala.

Debrie, Jean y Sandrine De Guio (2004), "Interfaces portuaires et compositions spatiales: instabilités africaines", en Autrepart, vol. 4, núm. 32, pp. 21-36

Foucher, Michel (1988), Fronts et frontières. Un tour du monde géopolitique, París: Fayard.

Fourny, Marie Christine (2005), "De la frontière naturelle à la nature comme lien transfrontalier. Du rôle et de la place de l'environnement et du milieu dans les coopérations transfrontalières", en Hélène Velasco-Graciet, y Christian Bouquet (eds.), Tropisme des frontières, Approche multidisciplinaire, t. 1. París: L'Harmattan, pp. 97-116.

Girot, Pascal y Carlos Granados Chaverri (1997), "La cooperación transfronteriza y los nuevos paradigmas de la integración centroamericana”, en Bovin, Philippe (ed.), Las fronteras del Istmo: fronteras y sociedades entre el sur de México y América Central, México: CEMCA, pp. 289-310.

Granados Chaverri, Carlos (2000), El mar, la nueva frontera centroamericana, San José de Costa Rica: Fundación para la Paz y la Democracia/Universidad de Costa Rica.

Groupe de Recherches Interfaces (2008), "L'interface : contribution à l'analyse de l'espace géographique", en L'Espace Géographique, vol. 3, t. 37, pp. 193-207.

Herrera Cáceres, Roberto (1974), Estatutojurídicode labahía de Fonseca y régimen de sus aguas adyacentes, Tegucigalpa: UNAH.

Jalkh, Gustavo (1995), Le statut juridique du Golfe de Fonseca, (tesis de doctorado en Derecho Público), Université Paris I, París.

Lampin-Maillet, Corinne et al. (coords.) (2010), Géographie des interfaces. Une nouvelle vision des territoires. París: Quae.

Lecoquierre, Bruno (2010), Différencier et échanger : les interfaces dans l'espace géographique, (tesis de habilitación), Université du Havre, Francia.

Lerner, Amy M. y Hallie Eakin (2011), "An obsolete dichotomy? Rethinking the rural-urban interface in terms of food security and production in the global south", en The Geographical Journal, vol. 177, núm. 4, diciembre, pp. 311-320.

"Marges et interfaces", número temático (actas de coloquio), revista Mosella, vol. 28 núms. 3-4, marzo 2006.

Matul Romero, Daniel (2007), "Vecindad, cooperación y confianza mutua: una revisión de las prácticas en las fronteras de Centroamérica", en Revista Centroamericana de Ciencias Sociales, vol. IV, núm. 1, julio, pp. 77-114.

Medina, Lucile (2009), "Les frontières de l'isthme centraméricain, de marges symboliques à des espaces en construction", en Espaces et Sociétés, núm. 138, pp. 35-50.

Medina, Lucile y Tania Rodríguez (2011), "Dinámicas de cooperación transfronteriza alrededor de la gestión ambiental de la cuenca del río Sixaola (Costa Rica/ Panamá)", en Aqua-LAC, Journal of the International Hydrological Programme for Latin America and the Caribbean (UNESCO), vol. 3, núm. 2, pp. 115-126.

Nelken-Terner, Antoinette (1997), "Las fronteras del Istmo centroamericano y el derecho internacional marítimo", en Bovin, Philippe (coord.), Lasfronteras del Istmo: fronteras y sociedades entre el sur de México y América Central, México: CEMCA.

Nelles, Jen y Olivier Walther (2011), "Changing European borders: from separation to interface? An introduction", en Journal of Urban Research, núm. 6.

Ó Dochartaigh, Niall (2007), "Conflict, territory and new technologies: Online interaction at a Belfast interface", en Political Geography, vol. 26, núm. 4, mayo, pp. 474-491.

Planche, Jeanne y Philippe Lavigne Delville (2005), "L'Union Européenne et les sociétés civiles du Sud : du discours politique aux actions de coopération", en Autrepart, vol. 3 núm. 35, pp. 143-160.

Rouvière, Laetitia (2009), “ L'élaboration des politiques locales de développement en milieu rural. Le cas de la triple frontière 'aymara' (Bolivie-Chili-Pérou)", en Laetitia Rouvière (coord.), La gouvernance frontalière, quelles opportunités pour les acteurs locaux?, Francia: Institut de Recherche et de Débat sur la Gouvernance. 
Robinson, William (2003), Transnational conflicts. Central America, social change and globalization, Londres/Nueva York: Verso.

Santamaría, Ángela y Xavier Zúñigo (2005), "Bonne gouvernance contre auto-gouvernance? Conflits, alliances et résistances autour d'un programme européen de développement rural en Colombie", en Autrepart, vol. 3, núm. 35, pp. 57-72.

Schofield, Clive (ed.) (1994), World Boundaries, vol. 5 Londres/Nueva York: Routledge. 
Mapa 1. La region trinacional del Golfo de Fonseca
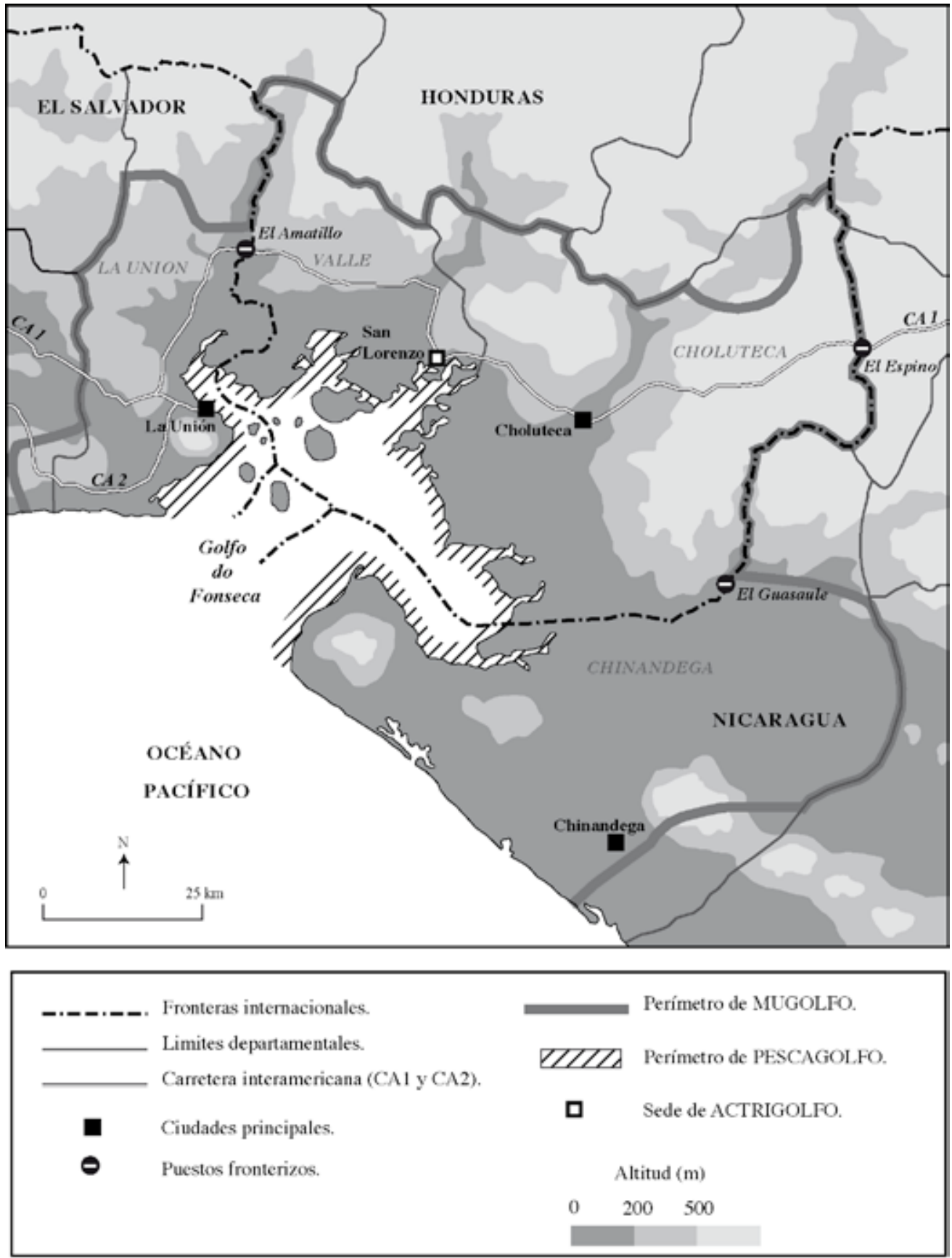

OS.C. - ART-Dev - UMR 5281 - CNRS / 2013

Fuente: Elaboración propia con base en trabajo de campo realizado entre 2010 y 2012. 